For exploring questions of competition and of island biogeography, the aquatic fauna of caves offers the advantages that the communities are relatively simple (usually fewer than 10 species) and that many replicates exist. Culver (Am. Nat. 110, 945; 1976) has used these facts in an elegant study of the aquatic communities in caves in river valleys in West Virginia, Tennessee and Virginia. Those who saw the movie Deliverance will wonder whether the NSF underwrote his insurance.

Although flatworms, molluscs, crayfish and salamanders are occasionally found, the macroscopic fauna in these caves is dominated by isopods and amphipods (two of the many orders of crustaceans), which account for more than $90 \%$ of the numbers and biomass in most caves. The streams in most Appalachian caves consist of a series of short riffles separated by larger pools. All the isopod and amphipod species prefer riffles to pools, partly because the rate of flow of food particles per unit of cross-sectional area in the stream is higher. The domain within a riffle is a patchy environment, with the habitable patches underneath rocks separated by uninhabitable areas where the creatures are exposed to the current. Since all the species are generalised detritus feeders, the main competition is simply for places to avoid the current.

Culver (Ecology 54, 102; 1973) has previously shown how, under these circumstances, the coefficients for interspecific competition can be estimated for laboratory studies using

\section{Caves as islands}

from Robert M. May

artificial streams. The competition coefficients, $\alpha$, thus obtained are in good agreement with the observed facts about aquatic cave communities: low $\alpha$-values are associated with species of different sizes (which tend to be under rocks of different sizes); intermediate $\alpha$-values are associated with species found in different riffles in the same cave; and species never found in the same cave have high $\alpha$ values. A more detailed analysis of the data available from the laboratory studies shows that communities with three species should be stable and resistant to invasion, which accords with the field observations.

The discussion, based on competition for habitat, explains many of the features of the aquatic cave communities, and suggests most caves could support three species. The average number of species per cave, is, however, more typically two (2.1 species per cave for the Powell River sites, from a total candidate pool of 4 species, and 2.5 species per cave from Greenbriar River sites, from a total pool of 6 species). Culver attributes this to the caves being, in effect, islands. As a consequence, the average number of species per cave is not simply the maximum number consistent with competitive interactions, but rather is some lesser number set by the interplay between extinction and immigration rates.

Such island biogeographical con- siderations can be worked out in some detail. The upshot of the processes of immigration and local extinction is that any one cave will tend to have fewer species (typically two), and the entire region to have more species (typically four to six), than would be the case if one had the maximum number of stably coexisting species (typically three) in one large cave. This re-echoes a theme first sounded theoretically by Skellam (Biometrika 38, 196; 1951) and Hutchinson ( $\mathrm{Am}$. Nat. 95, 137; 1961), with subsequent elaborations by Horn and MacArthur (Ecology 53, 749; 1972) and by Levins and Culver (Proc. natn. Acad. Sci. U.S.A. 68, 1246; 1971). For organisms of this kind, a conservation programme aimed at preserving maximum species diversity would probably be better served by several small refuges rather than one large one (Simberloff \& Abele Science 191, 285; 1976). More generally, the optimum pattern for allocation of conservation areas must depend on the life-history details of the target organism.

Culver concludes by emphasising that aquatic cave communities are basically simpler than terrestrial ones. The available evidence indicates, for terrestrial cave communities, that predation rather than competition may play the dominant role, that seasonal changes are more pronounced, and that the available pool of species is greatly affected by local geography. As Culver puts it: 'If you have seen one [type of] cave community, you have not seen them all.'

Robert M. May is the Class of 1877 Professor of Zoology at Princeton University these arrays with respect to the loops varies widely, both within and between species. In many cases a single array occupies all or most of a loop, but some contain a single array which does not span the entire loop, and may occupy as little as $30 \%$ (K. E. Scheer, Heidelberg). Other loops contain two arrays, sometimes with the same, and sometimes with opposite polarity. Still others contain multiple arrays-5-10\% of the loops in Triturus, $\sim 50 \%$ in the axolotl. When multiple arrays have the same polarity, they may of course represent a single transcription unit with the intervention of one or more processing steps. Where the polarities are opposite this is less likely, and it seems most reasonable to assume for the present that a loop may contain more than one transcription unit.

\section{Organisation}

This relates to the relationship between the loops and the axial chromomeres. The proportion of the DNA present in loops is difficult to measure exactly, but it clearly varies widely from one organism to another. In Triturus it is put at between 5 and $10 \%$, in Pleurodeles and the axolotl at $\sim 50 \%$. At one time it was speculated that each loop is progressively spooled out from one chromomere (the exit strand) and simultaneously gathered up into the other (the re-entrant strand). This is no longer widely believed, and the loops are thought to be more or less constant. The best evidence for this view comes from Triturus where it has been shown that the total nuclear RNA corresponds to a transcript of about $5 \%$ of the genome-roughly the proportion of the genome found in loops (D. B. Malcolm, St. Andrews). This is not, of course, a rigorous argument.

Techniques have been developed to pick out the same loop or set of loops in different preparations, and in several cases the functions of particular loops have been positively identified. Of the various methods, some depend for their specificity on nucleic acid reassociation, others apparently on the recognition of nucleic acids by proteins, and yet others on antigen-antibody interactions.

Histone genes from a number of echinoderms have been cloned in bacterial plasmids and bacteriophage. Nick-translated cloned DNA has been used to search for the histone genes of $T$. c. carnifex (Callan). Of the five biggish loops in the sexually heteromorphic region of chromosome $I$, three become labelled with these probes in conditions in which the loopassociated RNA is detected. Four other sets of loops on chromosomes VI(2), $X$ and XI are also labelled. Interestingly, differences were observed between the loop-sets of heterozygous bivalents in two cases, and minor differences between different newts are seen. A loop that is not otherwise visible, presumably because it carries little matrix, is visualised 